\title{
Assessment of superior cavo-pulmonary anastomoses (Glenn shunt) by cardiac magnetic resonance imaging in comparison with multi-slice computed tomography
}

\author{
Marwa Ali Gamal El-Deen ${ }^{1 *} \mathbb{D}$, Ahmed Samir Ibrahim', Emad H. Abdeldayem', Remon Zaher Elia and \\ Soha Romeih ${ }^{2}$
}

\begin{abstract}
Background: Multi-slice computed tomography (MSCT) angiography is the gold standard imaging modality to evaluate the patency of Glenn shunt and the presence of veno-veno collaterals. The goal of this study is to evaluate the ability of two cardiac magnetic resonance imaging (MRI) techniques to assess the patency of Glenn shunt and the presence of veno-veno collaterals compared to MSCT angiography.

Results: Patients with Glenn shunt had MSCT angiography and cardiac MRI using two techniques: TWIST (Timeresolved angiography With Stochastic Trajectories) and the three-dimensional (3D) post-contrast whole heart techniques. MSCT angiography and cardiac MRI images were post-processed for quantitative and qualitative assessment of Glenn shunt and veno-veno collaterals. Our study included 29 patients (17 male, 59\%) with Glenn shunt, the median age was 22 years (range 3-36 years). 3D post-contrast whole heart images give similar results compared to MSCT angiography results in the evaluation of Glenn shunt and veno-veno collaterals, $100 \%$ agreement in Glenn shunt visualization and agreement was $86.2 \%$ in the detection of veno-veno collaterals with a perfect agreement $($ kappa $=1)$ as regards their proximal connection to superior vena cava $($ SVC). While TWIST showed lower agreement compared to MSCT angiography results, $87.5 \%$ agreement in Glenn shunt visualization and agreement was $68.9 \%$ in the detection of veno-veno collaterals with poor agreement $(\mathrm{kappa}=0.266)$ as regards their proximal connection to SVC.
\end{abstract}

Conclusions: 3D post-contrast whole heart MRI images have similar results as MSCT angiography in the evaluation of superior cavo-pulmonary anastomosis and can be a good and safer alternative to MSCT angiography.

Keywords: MSCT, TWIST, 3D post-contrast whole heart, Glenn shunt

\section{Background}

Single ventricle pathophysiology is one of the most challenging congenital heart diseases (CHD) [1]. Cavopulmonary anastomosis (Glenn, Kawashima, and Fontan procedures) is the palliative surgical treatment for

\footnotetext{
*Correspondence: marwagamal2014@gmail.com

${ }^{1}$ Radiology Department, Faculty of Medicine, Ain Shams University, Cairo, Egypt

Full list of author information is available at the end of the article
}

those group of patients. The aim of this palliative surgical methods is the deviation of the systemic venous return into the pulmonary arterial circulation without passing through a ventricle to maximize systemic blood oxygenation and to maintain the functional capacity of the systemic ventricle [2]. The Glenn shunt (in which superior vena cava blood is rerouted directly into the pulmonary circulation) is usually the first stage of cavo-pulmonary anastomosis and is usually performed 
in early childhood. The Fontan procedure (in which inferior vena cava blood is redirected into pulmonary circulation) is the completion of the cavo-pulmonary anastomosis and is performed later in life. In many developing countries, many adult patients with Glenn shunt have no access for completion of cavo-pulmonary anastomosis [3]. Development of veno-veno collaterals is a common consequence for having Glenn shunt for a long time. Recanalization of the azygous vein is the most common type of veno-veno collaterals. However, numerous other venous channels may reopen under increased filling pressure. This, sequentially, causes desaturation and impaired daily performance in those patients and requires embolization or ligation [4]. Having a proximal connection with superior vena cava (SVC) or brachiocephalic vein, give the option to be closed by coil or devise percutaneously which leads to significant improvement of patients' condition [5]. Therefore, adult patients with Glenn shunt need followup for assessment of Glenn shunt patency and development of veno-veno collaterals. MSCT angiography is considered the gold standard imaging modality in the evaluation of the patient with Glenn shunt. However, high radiation dose and overlapping data acquisition is still the major inherent limitation, especially for children [6]. Cardiac magnetic resonance imaging has superior tissue contrast and spatial resolution in multiple planes without ionizing radiation [7]. Also, cardiac MRI is superior in its inherent capability to identify intra-cardiac anatomical, functional, and structural abnormalities as well as postoperative complications following surgical procedures for congenital heart disease [8].

Time-resolved magnetic resonance angiography (MRA) sequences, known under some acronyms, one of them is TWIST, obtain a series of images displaying the passage of the contrast bolus. TWIST is very important in thoracic imaging as it allows venous mapping of the central veins of the chest and facilitates detection of stenosis and vascular anomalies and evaluation of the collateral flow to further define hemodynamic significance [9].

The 3D post-contrast whole heart approach with respiratory navigator gating and electrocardiogram (ECG) triggering enables comprehensive evaluation of thoracic vasculature through detailed morphological information in patients with CHD [10].

The aim of our study is to evaluate ability of two cardiac MRI techniques, TWIST, and 3D post-contrast whole heart, in the evaluation of superior cavo-pulmonary anastomoses regarding patency of the Glenn shunt and presence of veno-veno collaterals in comparison with MSCT angiography findings.

\section{Methods}

All patients with Glenn shunt who were referred to our department in the period between March 2019 and March 2020 were included. All patients had MSCT angiography and two cardiac MRI techniques (TWIST and 3D post-contrast whole heart techniques) for evaluation of the patency of Glenn shunt and presence of veno-veno collaterals [11, 12].

Inclusion criteria: (1) When the Glenn shunt could not be evaluated by Echocardiography or (2) There was a reversal of flow over the shunt by echocardiography or (3) When patient recent complaints of shunt failure like cyanosis. Our study was approved by the research ethic committee of the radiology department of our university.

Exclusion criteria: (1) Claustrophobia to MRI machine or (2) Patients with cochlear implant or metallic ocular foreign body or non-compatible implanted pacemaker or (3) Established renal failure with impaired hepatic function.

\section{MSCT angiography}

All MSCT angiography examinations were performed using a 128 dual source multi-slice SOMATOM scanner (Siemens, Erlangen, Germany). The examination was performed with retrospective electrocardiogram ECG gating and tube modulation to reduce the radiation dose. The tube current modulation technique has led to a considerable reduction in radiation dose $(60 \%$ dose reduction). Analysis was performed on the best phase with less artifacts either systolic phase or diastolic phase. The scanning parameters include detector collimation of 128 to $0.6 \mathrm{~mm}$, gantry rotation time of $330 \mathrm{~ms}$, and pitch of (0.3-0.4) adjusted according to heart rate. The tube potential was 100 to 120 kilovoltage peak (kvp). The rotation time was $0.28 \mathrm{~s}$, and its temporal resolution was $75 \mathrm{~ms}$. We have used a nonionic contrast medium (Ultravist 370 ), as $2 \mathrm{~mL} / \mathrm{kg}$ at a rate of $1-2.5 \mathrm{~mL} / \mathrm{s}$ followed by $15-20 \mathrm{~mL}$ saline.

\section{MRI examination}

All cardiac MRI examinations were performed using a $1.5 \mathrm{~T}$ Magnetom Aera (Siemens, Erlangen, Germany). All patients were examined in a supine position and headfirst. ECG leads and a phased-array cardiac coil were used. Two cardiac MRI techniques were used:

- A) TWIST: It was performed with a 3D spoiled gradient-recalled echo acquisition in the coronal orientation. It was performed after administration of $0.2 \mathrm{mmol} / \mathrm{kg}$ of gadoterate meglumine (Dotarem). The contrast agent was administered at a flow rate of $0.9 \mathrm{~mL} / \mathrm{s}$, followed by a $15-20-\mathrm{mL}$ saline flush at 
the same flow rate. Image acquisition was started just before contrast administration and continued after contrast agent injection. The scan parameters: Reptation time/time to echo (TR/TE: 2.52/0.87), flip angle $=2.9^{\circ}$, bandwidth $=610 \mathrm{~Hz} /$ pixel; field of view $($ FOV $)=450 \times 337 \mathrm{~mm}^{2}$ matrix $=256 \times 134$, phase resolution $70 \%$, slice resolution $64 \%$, temporal resolution $=3.87 \mathrm{~s}$, voxel size $=1.8 \times 1.8 \times 1.3 \mathrm{~mm}$.

- B) 3D post-contrast whole heart was performed after TWIST using an ECG-gated and respiratorytriggered 3D balanced steady-state free precession sequence with T2 preparation and fat suppression. Respiratory triggering was performed using a navigator placed on the right hemidiaphragm with a $5 \mathrm{~mm}$ displacement window. 3D imaging was performed in either the coronal plane or the oblique sagittal plane. The scan parameters: TR/TE 264/1.39, flip angle $88^{\circ}$, slice thickness 1.5, and slice/slab 112.

Imaging post-processing was performed on a workstation (View Forum, Philips Medical Systems, Best, The Netherlands). The multiplanar reformatting (MPR), maximum intensity projections (MIP), and 3D volumerendered technique (VRT) reconstructions were used to assess the Glenn shunt and veno-veno collaterals. The MSCT angiographic images as well as both cardiac MRI techniques have been analyzed for:

\section{(A) Glenn shunt assessment}

(1) Qualitative assessment: visualization, thrombosis (defines as hypodense filling defect were assessed.)

(2) Quantitative assessment: diameter of Glenn shunt was measured at the insertion point with the pulmonary artery, and stenosis was defined as $50 \%$ reduction in lumen compared to the proximal part.

(B) Veno-veno collaterals assessment
(1) Qualitative assessment: presence and connection to SVC or brachiocephalic vein,

(2) Quantitative assessment: number of collaterals and diameter measured at the widest part.

\section{Analysis of data}

The agreement between MSCT angiography and each cardiac MRI technique for all qualitative assessment was analyzed by calculating kappa. Kappa below 0.4 was a poor concordance, kappa between 0.4 and 0.75 was a moderate concordance, and kappa between 0.76 and 1 was a strong concordance [13]. The agreement between MSCT angiography and each cardiac MRI technique for all the quantitative assessment was analyzed by calculating the mean difference $\pm S D$, for Bland and Altman, and interclass correlation (ICC). ICC below 0.4 was a poor concordance, ICC between 0.4 and 0.75 was a moderate concordance, and ICC between 0.76 and 1 was a strong concordance [14]. $P<0.05$ was considered statistically significant.

\section{Results}

The study included 29 patients (17 male) with 32 Glenn shunts as there were 3 patients with bilateral Glenn shunts. The median age of patients was 22 years (range 3-36 years). All children tolerated the examination without sedation except one child (3-years old age) who was sedated by oral chloral hydrate $(75 \mathrm{mg} / \mathrm{kg})$.

(A) Glenn shunt assessment:

- Glenn patency, stenosis, and thrombosis:

There was $100 \%$ agreement between 3D post-contrast whole heart and MSCT angiography in visualization and detection of any stenosis or thrombosis of Glenn shunt as shown in Tables 1, 2 and 3. There is a strong agreement between MSCT angiography and 3D post-contrast whole heart in detection stenosis of Glenn shunt with kappa agreement $=1$.

Table 1 Glenn shunt visualization

\begin{tabular}{|c|c|c|c|c|c|}
\hline \multirow[t]{2}{*}{$N=32$} & \multicolumn{2}{|c|}{ Glenn visualization } & \multirow[t]{2}{*}{ Test value* } & \multirow[t]{2}{*}{$P$ value } & \multirow[t]{2}{*}{ Significance } \\
\hline & MSCT & $3 \mathrm{D}$ post-contrast whole heart & & & \\
\hline \multirow[t]{2}{*}{ Yes } & $32(100.0 \%)$ & $32(100.0 \%)$ & 0.000 & 1.000 & NS \\
\hline & MSCT & TWIST & Test value* & $P$ value & Significance \\
\hline Yes & $32(100.0 \%)$ & $28(87.5 \%)$ & 4.267 & 0.039 & S \\
\hline
\end{tabular}

P-value $>0.05$ : Non significant; P-value $<0.05$ : Significant; P-value $<0.01$ : Highly significant

*: Chi-square test 
Table 2 Glenn shunt stenosis

\begin{tabular}{|c|c|c|c|c|c|}
\hline & \multicolumn{2}{|c|}{ Stenosis of Glenn shunt } & \multirow[t]{3}{*}{ Test value* } & \multirow[t]{3}{*}{$P$ value } & \multirow[t]{3}{*}{ Significance } \\
\hline & MSCT & 3D post-contrast whole heart & & & \\
\hline & 32 & 32 & & & \\
\hline \multirow[t]{3}{*}{ Yes } & $1(3.1 \%)$ & $1(3.1 \%)$ & 0.000 & 1.000 & NS \\
\hline & MSCT & TWIST & Test value* & $P$ value & Significance \\
\hline & 28 & 28 & & & \\
\hline Yes & $0(0.0 \%)$ & $0(0.0 \%)$ & 0000 & 1.000 & NS \\
\hline
\end{tabular}

$\mathrm{P}$-value $>0.05$ : Non significant; $\mathrm{P}$-value $<0.05$ : Significant; $\mathrm{P}$-value $<0.01$ : Highly significant

*: Chi-square test

Table 3 Glenn shunt thrombosis

\begin{tabular}{|c|c|c|c|c|c|}
\hline & \multicolumn{2}{|c|}{ Thrombosis of Glenn shunt } & \multirow[t]{3}{*}{ Test value* } & \multirow[t]{3}{*}{$P$ value } & \multirow[t]{3}{*}{ Significance } \\
\hline & MSCT & $3 \mathrm{D}$ post-contrast whole heart & & & \\
\hline & 32 & 32 & & & \\
\hline \multirow[t]{3}{*}{ No } & $32(100 \%)$ & $32(100 \%)$ & 0.000 & 1.000 & NS \\
\hline & MSCT & TWIST & Test value* & $P$ value & Significance \\
\hline & 28 & 28 & & & \\
\hline No & $28(100 \%)$ & $28(100 \%)$ & 0.000 & 1.000 & NS \\
\hline
\end{tabular}

P-value $>0.05$ : Non significant; P-value $<0.05$ : Significant; P-value $<0.01$ : Highly significant

*: Chi-square test

There was $87.5 \%$ agreement between TWIST and MSCT angiography in Glenn shunt visualization as seen in Table 1. TWIST could not visualize 4 Glenn shunts due to (1) stealing of the contrast from the Glenn shunt via a mesh of multiple collaterals in one patient (2) improper timing of imaging acquisition in three patients due to improper contrast bolus timing.

The agreement was 100\% regarding detection of Glenn shunt stenosis and thrombosis, as shown in Tables 2 and 3.

- Glenn diameter: Mean diameter by MSCT angiography was $16.94 \pm 5.76 \mathrm{~mm}$, by 3D post-contrast whole heart was16.41 $\pm 5.05 \mathrm{~mm}$, and by TWIST was $14.64 \pm 5.25 \mathrm{~mm}$. Bland Altman figure (Fig. 1) showed a strong correlation between MSCT angiography and 3D post-contrast whole heart $(P$ value $<0.081)$, also a strong correlation between MSCT angiography and TWIST $(P$ value $<0.001)$ as seen in Fig. 2.

(B) Veno-veno collaterals assessment:
- The total number of veno-veno collaterals by MSCT angiography was 49 collaterals in 21 patients. The 3D post-contrast whole heart was able to detect 40 collaterals in 21 patients; therefore, the percentage of agreement was $86.2 \%$. The $3 \mathrm{D}$ post-contrast whole heart had missed 9 venoveno collaterals in five patients due to improper

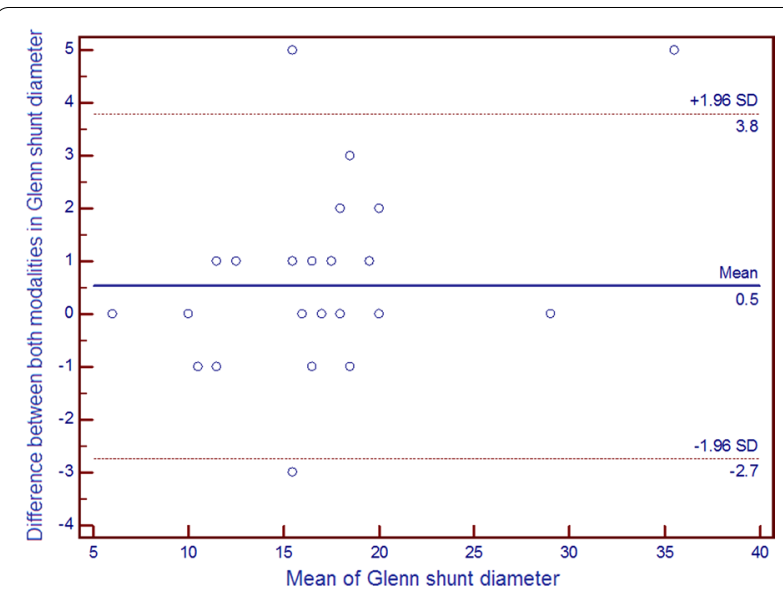

Fig. 1 Bland-Altman figure plotting the difference between MSCT angiography and 3D post-contrast whole heart regarding the diameter of Glenn 


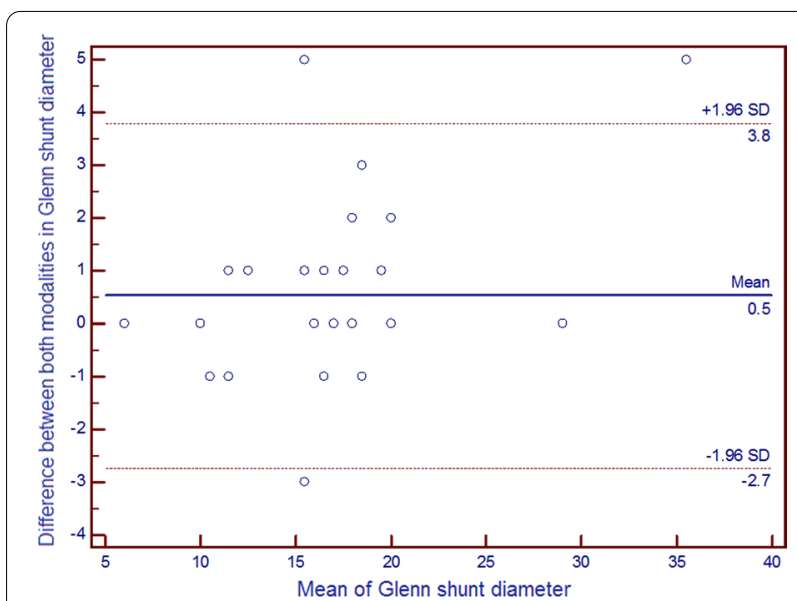

Fig. 2 Bland-Altman figure plotting the difference between MSCT angiography and TWIST regarding the diameter of Glenn image acquisition due to extra-premature beats. While TWIST was able to detect 20 collaterals in 12 patients, therefore the percentage of agreement was $68.9 \%$. Thirteen veno-veno collaterals were missed in eight patients due to severe kyphoscoliosis which makes tracing of the contrast very inaccurate, while 16 collaterals in ten patients were missed due to the small size, all of them are smaller than $4 \mathrm{~mm}$ in size.

- Size of veno-veno collaterals: Mean diameter by MSCT angiography was $6.93 \pm 3.40 \mathrm{~mm}$, by 3D post-contrast whole heart was $6.93 \pm 3.53 \mathrm{~mm}$ and by TWIST was $5.95 \pm 2.68 \mathrm{~mm}$. Bland Altman figure (Fig. 3) showed a strong correlation

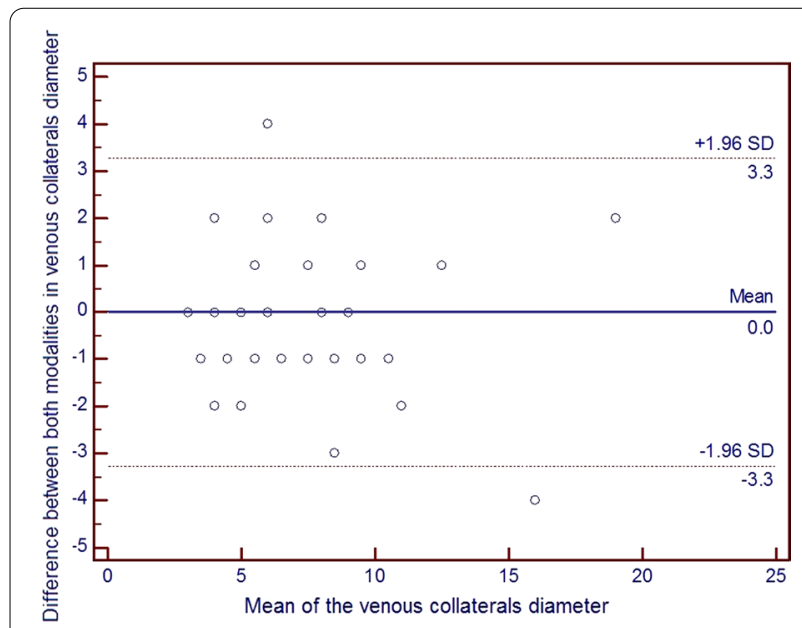

Fig. 3 Bland-Altman figure plotting the difference between MSCT angiography and 3D post-contrast whole heart regarding the size of collaterals

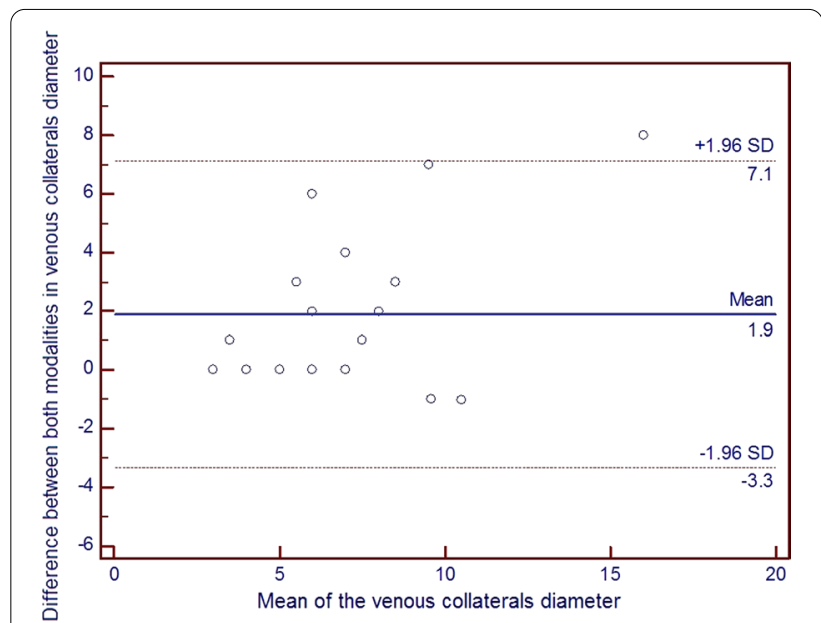

Fig. 4 Bland-Altman figure plotting the difference between MSCT angiography and TWIST regarding the size of collaterals

between MSCT angiography and 3D post-contrast whole heart $(P$ value $=1)$, also a strong correlation between MSCT angiography and TWIST $(P$ value $=0.006)$ as seen in Fig. 4 .

- Connection to SVC/brachiocephalic vein: MSCT angiography showed that 14 collaterals were connected to SVC/or brachiocephalic vein as seen in Fig. 5. The 3D post-contrast whole heart detected them all with kappa agreement $=1$, while TWIST detected four collaterals connected to SVC/or brachiocephalic vein as seen in Fig. 6 with lower kappa agreement $=0.266$. TWIST did not detect ten collaterals connected to SVC in nine patients that may be attributed to the small size of collaterals and blurring of the image.

\section{Discussion}

Our study evaluated the ability of two cardiac MRI techniques, TWIST, and 3D post-contrast whole heart, in the evaluation of patients with superior cavo-pulmonary anastomoses regarding patency of Glenn shunt and presence of veno-veno collaterals compared to MSCT angiography findings. We found that $3 \mathrm{D}$ post-contrast whole heart has a statistically significant agreement in qualitative and quantitative assessment for both Glenn shunt and veno-veno collaterals. While TWIST showed lower agreement due to the improper timing of contrast bolus tracking, either due to arrhythmia, improper movement, kyphoscoliosis, or small size of the veno-veno collaterals $(<4 \mathrm{~mm}$ in size) . 

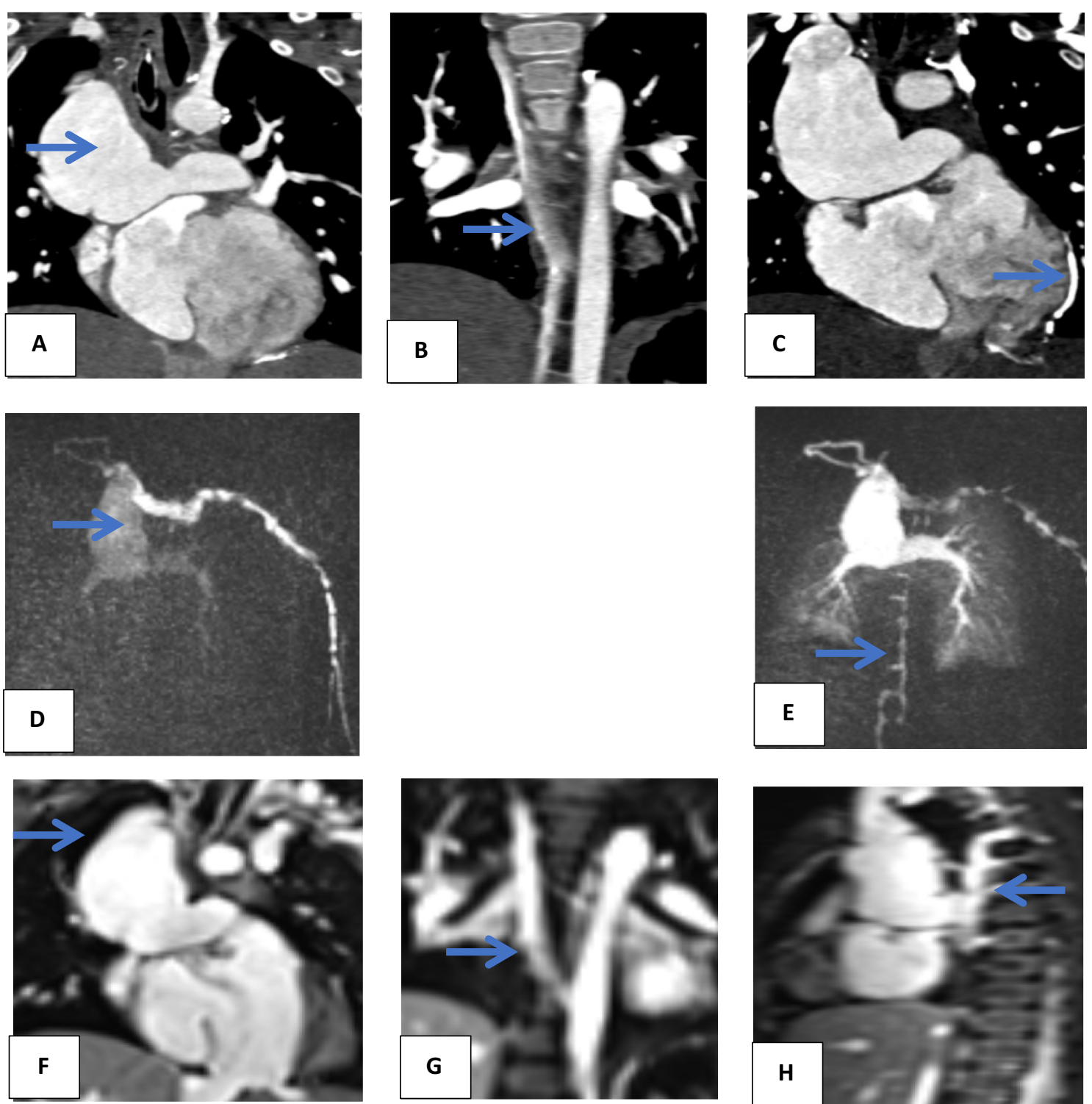

Fig. 5 A 3-year-old male child, known to have unbalanced CAVC and TAPVR treated with PA band and right-sided Glenn shunt A MSCT angiography coronal view showed aneurysmal dilation of right-sided Glenn that was connected to the right pulmonary artery. B MSCT angiography coronal view showed patent azygous vein. C MSCT angiography coronal view showed venous collateral along the left hemidiaphragm draining into IVC. D TWIST showed aneurysmal dilation of right-sided Glenn. E TWIST showed early rapid filling of paravertebral plexuses. F 3D post-contrast whole heart coronal view revealed aneurysmal dilation of right-sided Glenn that was connected to right pulmonary artery. G 3D post-contrast whole heart coronal view revealed patent azygous vein. $\mathbf{H}$ 3D post-contrast whole heart sagittal view revealed azygous vein connected to the paravertebral venous plexus

\section{MSCT angiography}

MSCT angiography is the gold standard imaging modality in the evaluation of cavo-pulmonary anastomosis and related complications such as thrombosis, stenosis, and development of veno-veno collaterals. The main advantages of MSCT angiography are the short acquisition time, high spatial resolution, and ability to image patients with various metallic stents, pacemakers, and defibrillators [2].

It has been reported that MSCT angiography is an accurate and reliable noninvasive examination in the assessment of morphological changes in patients with Glenn shunt, regarding visualization, stenosis, and thrombosis $[8,15]$. 

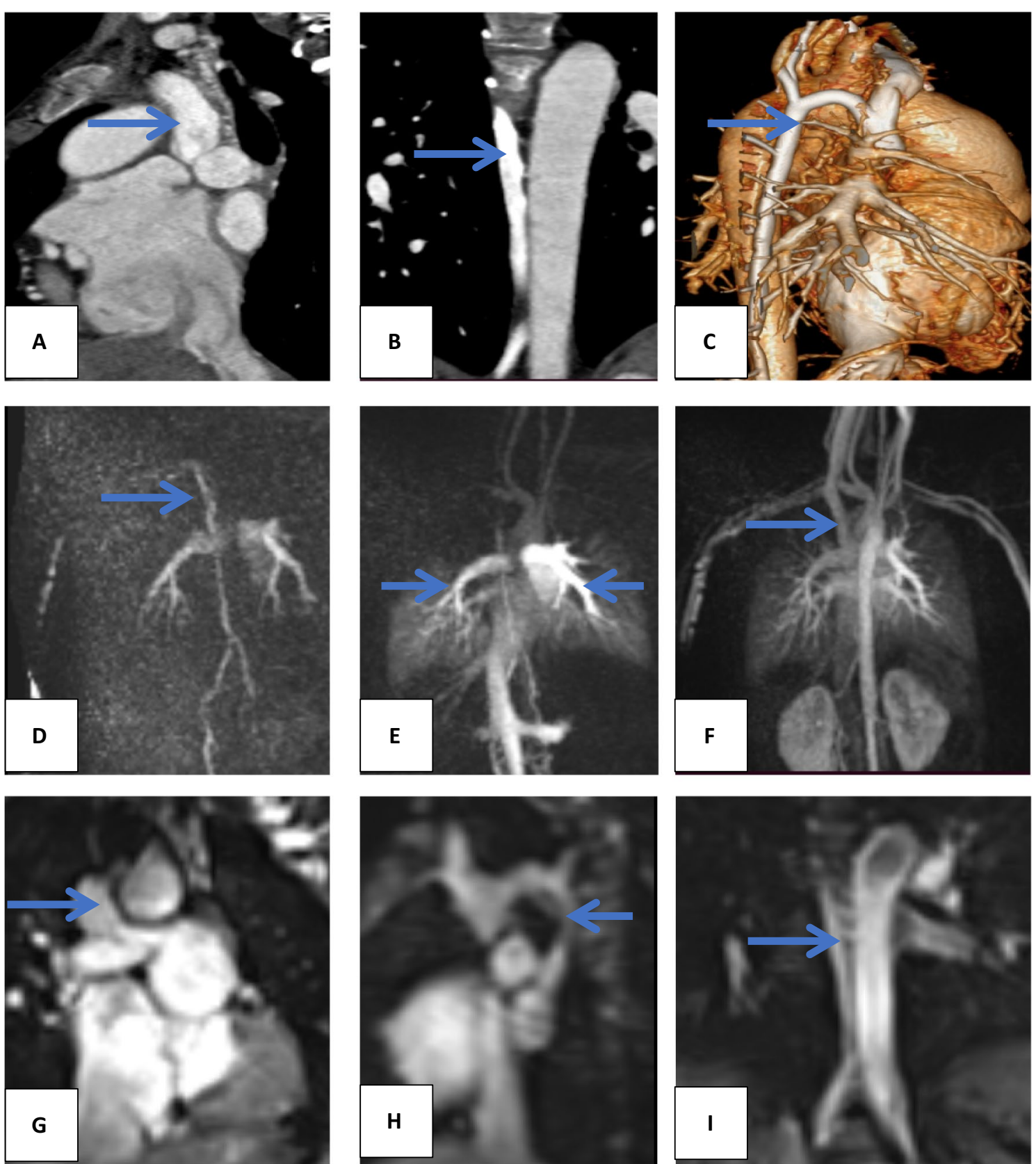

Fig. 6 An 11-year-old male with a history of D-TGA, LVOTO and ASD treated with right -sided Glenn shunt. A MSCT angiography sagittal view showed patent right-sided Glenn that was connected to the right pulmonary artery. B MSCT angiography coronal view showed patent azygous vein. C MSCT angiography -VRT showed azygous vein connected to SVC. D TWIST showed azygous vein and faint both pulmonary arteries on the first pass. E TWIST showed well visualized both pulmonary arteries and aorta in the next dynamic sequence. F TWIST showed lastly patent right-sided Glenn with no stenosis or thrombosis. G 3D post-contrast whole heart that showed patent right-sided Genn shunt. H 3D post-contrast whole heart that showed patent azygous vein which connected to SVC. I 3D post-contrast whole heart that showed patent azygous vein

The major disadvantage to MSCT angiography is the use of ionizing radiation. Despite technological progresses that have decreased radiation exposure, for young patients that will require serial imaging, the cumulative radiation dose remains a concern. MSCT angiography always requires the use of iodinated contrast, which can be an issue for patients with chronic renal disease or contrast allergies. Also, technical expertize is essential, as the timing of the scan must be optimized for maximum contrast enhancement of the 
structure of interest as failure to properly address these structures may lead to unnecessary risk to the patient from radiation and contrast exposure with a low likelihood of answering the clinical question. Moreover, MSCT angiography requires ECG gating and irregular rhythms can make for challenging images [16].

\section{D post-contrast whole heart sequence}

3D post-contrast whole heart technique is a keystone cardiac MRI technique in the evaluation of patients with CHD. The comprehensive assessment of the thoracic vasculature it presents is uniquely suited to give detailed morphological information in CHD. It offers significant advantages, no ionizing radiation; the ability to be run free-breathing; and higher signal-to-noise ratio and isotropic voxel resolution for multiplanar reformatting assessment. It is now frequently used and has a proven utility for diagnostic imaging in patients with CHD [17].

\section{TWIST}

TWIST is a technique that creates a sequential fast series of multiplanar images with a good spatial resolution during the passage of intravenous contrast medium. It generates a series of imaging datasets at different time points of the same intravenous contrast medium bolus (unenhanced, pulmonary venous, systemic arterial, early systemic venous, and late systemic venous) allowing comprehensive assessment of the arterial and venous evaluation of clinical service [18].

Our results agree with Huf et al. in 2020 that found TWIST although being a very fast sequence but still suffered from loss of signal, resulting in a low SNR as well as blurring of small vessels [19]. However, TWIST provides a rapid overview of the status and patency of vascular structures. With only small contrast material doses, TWIST may be utilized to detect intra- and extracardiac shunts, to evaluate dynamic vascular anatomy in thoracic vascular disease [18].

In contrast to our results, the results from Michael et al. in 2007 found that Glenn shunt patency was better using TWIST. However, the number of patients was only six patients which make the comparison very difficult [20].

To the best of our knowledge, no available published data regarding the assessment of Glenn shunt by 3D post-contrast whole heart sequence. However, results published in 2008 by Tomasian et al. demonstrated that $3 \mathrm{D}$ whole heart sequence is optimal for evaluating the SVC as compared with MR angiography [21].

Also, results from Sonavane et al. in 2015 confirmed that $3 \mathrm{D}$ whole heart images in the axial and coronal planes are useful in evaluating the SVC lumen and wall and adjacent mediastinal structures [22].

According to the obtained results, the accuracy of this sequence in the assessment of superior cavo-pulmonary anastomosis could be attained with the better spatial resolution of the 3D post-contrast whole heart in addition to the lack of need to trace the contrast and optimization of the imaging time.

To the best of our knowledge, our study is the first study to compare MSCT angiography with two cardiac MRI techniques TWIST and 3D post-contrast whole heart in the evaluation of superior cavo-pulmonary anastomosis and systemic veno-venous collaterals based on quantitative and qualitative assessment.

The limitations for this study were the relatively small number of subjects and therefore further studies with a large patient population are warranted. Additionally, the study was done in a single center.

\section{Conclusions}

Although MSCT angiography is still the gold standard imaging modality for the assessment of superior cavo-pulmonary shunt and veno-veno collaterals, 3D post-contrast whole heart sequence is a good and safer alternative imaging modality compared to MSCT angiography. While TWIST results are much lower in accuracy compared to MSCT.

\section{Abbreviations}

MSCT: Multi-slice computed tomography; MRI: Magnetic resonance imaging; TWIST: Time-resolved angiography With Stochastic Trajectories; 3D: Threedimensional; SVC: Superior vena cava; CHD: Congenital heart disease; MRA: Magnetic resonance angiography; ECG : Electrocardiogram; Kvp : Kilovoltage peak; TR/TE : Reptation time/time to echo; FOV : Field of view; MPR : Multiplanar reformatting; MIP : Maximum intensity projections; VRT : Volume-rendered technique; ICC : Interclass correlation; CAVC : Complete atrioventricular canal; TAPVR: Total anomalous pulmonary venous return; PA : Pulmonary artery; IVC : Inferior vena cava; D-TGA : Dextro-transposition great arteries; LVOTO : Left ventricle outflow tract obstruction; ASD : Atrial septal defect.

\section{Acknowledgements}

The authors would like to thank Dr. Wesam El-Mozy, Dr. Fatma El-Kafrawy, Dr. Mahmoud Naseem, Dr. Ahmed El-Mogy, Dr. Al Shaimaa Ahmed and Dr. Mahmoud Salah for their contribution.

\section{Authors' contributions}

All authors contributed to the study conception and design. Material preparation, data collection and analysis were done by MG and SR. The first draft of the manuscript was written by MG and authors commented on previous versions of the manuscript. All authors read and approved the final manuscript.

\section{Funding}

The author had no fund for this research.

Availability of data and materials

All data are available. 


\section{Declarations}

Ethics approval and consent to participate

The protocol was checked and approved by local ethics committee of the radiology department, Ain Shams University. All patients had been given written consents to participate in this work.

\section{Consent for publication}

All patients had given their written consents for publication of this work.

\section{Competing interests}

The authors declare that they have no competing interests.

\section{Author details}

${ }^{1}$ Radiology Department, Faculty of Medicine, Ain Shams University, Cairo, Egypt. ${ }^{2}$ Director of Advanced Cardiac Imaging at Aswan Heart Center (Magdi Yacoub Foundation), Cardiology Department, Faculty of Medicine, Tanta University, Tanta, Egypt.

Received: 13 October 2021 Accepted: 6 December 2021 Published online: 15 December 2021

\section{References}

1. Kutty S, Rathod HR, Danford DA et al (2016) Role of imaging in the evaluation of single ventricle with the Fontan palliation. Heart 102:174-183. https://doi.org/10.1136/heartjnl-2015-308298

2. Hong HS, Kim MY, Lee C et al (2017) CT and MRI evaluation of the Fontan pathway: pearls and pitfalls. Cardiovasc Innov Appl (CVIA) 1:133-145. https://doi.org/10.22468/cvia.2016.00157

3. Tomasian A, Malik S, Shamsa K et al (2009) Congenital heart diseases: post-operative appearance on multi-detector CT-a pictorial essay. Eur Radiol 19:2941-2949. https://doi.org/10.1007/s00330-009-1474-7

4. Mahani MG, Agarwal P, Rigsby C et al (2016) CT for assessment of thrombosis and pulmonary embolism in multiple stages of singleventricle palliation: challenges and suggested protocols. Radiographics 36:1273-1284. https://doi.org/10.1148/rg.2016150233

5. McElhinney DB, Mohan Reddy V, Hanley FL et al (1997) Systemic venous collateral channels causing desaturation after bidirectional cavopulmonary anastomosis: evaluation and management. J Am Coll Cardiol 30:817-824. https://doi.org/10.1016/s0735-1097(97)00223-4

6. Heinemann M, Breuer J, Steger $V$ et al (2001) Incidence and impact of systemic venous collateral development after Glenn and Fontan procedures. Thorac Cardiovasc Surg 49:172-178. https://doi.org/10. 1055/s-2001-14339

7. Zahr RA, Kirshbom PM, Kopf GS et al (2016) Half a century's experience with the superior cavopulmonary (Classic Glenn) shunt. Ann Thorac Surg 101:177-182. https://doi.org/10.1016/j.athoracsur.2015.08.018

8. Ji X, Zhao B, Cheng Z et al (2014) Low-dose prospectively electrocardiogram-gated axial dual-source CT angiography in patients with pulsatile bilateral bidirectional Glenn shunt: an alternative noninvasive method for postoperative morphological estimation. PLoS ONE 9:e94425. https://doi. org/10.1371/journal.pone.0094425

9. Saraya S, Woodard P, Bhalla S et al (2020) Cardiac MRI in the evaluation of postoperative congenital heart disease and complications. Egypt J Radiol Nucl Med 51:133. https://doi.org/10.1186/s43055-020-00250-x

10. Leschka S, Oechslin E, Husmann L et al (2007) Pre- and postoperative evaluation of congenital heart disease in children and adults with 64-section CT. Radiographics 27:829-845. https://doi.org/10.1148/rg.273065713

11. Kim CY, Merkle EM (2008) Time-resolved MR angiography of the central veins of the chest. AJR 191:1581-1588. https://doi.org/10.2214/AJR.08. 1027

12. Valsangiacomo Buechel ER, Grosse-Wortmann L, Fratz S et al (2015) Indications for cardiovascular magnetic resonance in children with congenital and acquired heart disease: an expert consensus paper of the Imaging Working Group of the AEPC and the Cardiovascular Magnetic Resonance Section of the EACVI. Eur Heart J Cardiovasc Imaging 16:281-297. https:// doi.org/10.1093/ehjci/jeu129
13. Fleiss $J L$ (1981) Statistical methods for rates and proportions, 2nd edn. Wiley, New York, pp 350-354

14. Koch GG (1999) Intraclass correlation coefficient. In: Kotz S, Johnson NL (eds) Encyclopaedia of statistical sciences. Wiley, New York, pp 213-217

15. Patel LD, Rana RY, Sheth MM et al (2019) MSCT imaging of various shunts and grafts in post-operative cases of congenital heart diseases. Int J Res Med Sci 7:860-865. https://doi.org/10.18203/2320-6012.ijrms20190937

16. Bonnichsen C, Ammash N (2016) Choosing between MRI and CT imaging in the adult with congenital heart disease. Curr Cardiol Rep 18:45. https:// doi.org/10.1007/s11886-016-0717-x

17. Greil G, Tandon A, Vieira SM et al (2017) 3D whole heart imaging for congenital heart disease. Front Pediatr 5:36. https://doi.org/10.3389/fped. 2017.00036

18. Armstrong L, Rodrigues JCL, Lawton CB et al (2016) Application of TWIST MR angiography to aid successful central venous access in challenging patients: initial single-centre experience. Clin Radiol 71:1104-1112. https://doi.org/10.1016/j.crad.2016.04.005

19. Huf VI, Fellner C, Wohlgemuth WA et al (2020) Fast TWIST with iterative reconstruction improves diagnostic accuracy of AVM of the hand. Sci Rep 10:16355. https://doi.org/10.1038/s41598-020-73331-6

20. Michael F, Roya S, Howard D et al (2007) Juvenile and adult congenital heart disease: time-resolved 3D contrast-enhanced MR angiography. Radiology 244:399-410. https://doi.org/10.1148/radiol.2442061045

21. Tomasian A, Lohan DG, Laub G et al (2008) Non-contrast 3D steady state free precession magnetic resonance angiography of the thoracic central veins using nonselective radiofrequency excitation over a large field of view: initial experience. Investig Radiol 43:306-313. https://doi.org/10. 1097/RLI.0b013e31816be927

22. Sonavane KS, Milner MD, Singh PS et al (2015) Comprehensive imaging review of the superior vena cava. Radiographics 35:1873-1892. https:// doi.org/10.1148/rg.2015150056

\section{Publisher's Note}

Springer Nature remains neutral with regard to jurisdictional claims in published maps and institutional affiliations.

\section{Submit your manuscript to a SpringerOpen ${ }^{\circ}$ journal and benefit from:}

- Convenient online submission

- Rigorous peer review

- Open access: articles freely available online

- High visibility within the field

Retaining the copyright to your article

Submit your next manuscript at springeropen.com 